\title{
Keratinizing Dentigerous Cyst - An Unusual Entity-A Rare
}

\section{Case Report}

\author{
Sarvesh Vijay ${ }^{1 *}$, Yogesh T. L ${ }^{2}$, AnjaniK Jha ${ }^{3}$, Nonitha.S ${ }^{4}$ \\ ${ }^{1}$ Department of Oral \& Maxillofacial Pathology, Sri Rajiv Gandhi College of Dental Sciences \& \\ Hospital, Cholanagar, Hebbal, Bangalore, Karnataka, India. \\ E-mail: sarvesh.rdc@gmail.com
}

\begin{abstract}
2Professor, Department of Oral \& Maxillofacial Pathology, Sri Rajiv Gandhi College of Dental Sciences \& Hospital, Cholanagar, Hebbal, Bangalore, Karnataka, India.
\end{abstract}

${ }^{3}$ Associate professor, Major SD Singh Medical College, and Hospital, Farrukhabad, (U.P), India.

${ }^{4}$ Senior Lecturer, Department of Oral \& Maxillofacial Pathology, Sri Rajiv Gandhi College of Dental Sciences and Hospital, Cholanagar, Hebbal, Bangalore, Karnataka, India.

\section{ABSTRACT}

Background: A dentigerous cyst or follicular cyst is one of the forms of odontogenic cysts. It encloses the crown of an un-erupted tooth by the expansion of its follicle within the follicular space. It is normally painlessly discovered during the routine radiographic examination. Importance of keratinization in odontogenic cysts is still a mystery as it is a very rare entity. This article reports a case of keratinizing dentigerous cyst associated with a mandible.

Case Presentation: A 50-year-old male patient with a chief complaint of swelling and pain in the right side of the lower jaw for 4 months. The lesion was provisionally diagnosed as OKC or dentigerous cyst after the clinical and radiographic examination. Treatment consisted of surgical excision and the excised specimen was sent for histopathological examination which confirmed the findings of a keratinizing dentigerous cyst.

Conclusions: Only a few cases of keratinizing dentigerous cyst has been reported in the literature so far to the best of our knowledge. Early recognition and intervention play a key role in its management due to its malignant transformation and potential for recurrence.

Keywords: Dentigerous cyst, Keratinizing, Odontogenic, Mandible.

\section{Introduction}

The most common non-inflammatory odontogenic cyst of the oral cavity is the dentigerous cyst. ${ }^{[1]}$ Scultet in the year 1671, first of all, described the jaw cysts as the liquid tumors. Paget in the year 1851 first of all coined the term dentigerous cyst. The word dentigerous means tooth bearing. ${ }^{[2] I t}$ develops by the accumulation of fluid within the follicular space of an unerupted tooth after its crown has fully formed. ${ }^{[3]}$ Dentigerous cysts can be either intra-follicular i.e accumulation of the fluid either between the reduced enamel epithelium and the enamel or within the enamel organ itself or it may be extra-follicular i.e foci of enamel hypoplasia. ${ }^{[4]}$ Dentigerous a cyst is the second most common odontogenic cyst, next to a radicular cyst. The most involved teeth include the third molars, the canines, and the second premolars. ${ }^{[5]} \mathrm{About} 70 \%$ of the cases occur in the mandible while maxilla comprises of about only $30 \%$ of the cases. ${ }^{[6]}$ It is more commonly seen in whites than in blacks, and according to Browne and Killey 1982, it is more commonly seen in males as compared to females (2:1). Gnepp 2001 stated that it may occur at 
any age but it is most commonly found in young adults.[7] The Keratinizing dentigerous cyst is a histopathological variant of a dentigerous cyst which is not encountered commonly, and only a few cases have been reported in the literature so far. The present report focuses on a case of an unusual histological variant of an odontogenic cyst, the keratinizing dentigerous cyst with a literature review.

\section{Case Report}

A 50-year-old male patient reported to the Department of Oral and Maxillofacial Surgery, Major SD Singh Medical College and Hospital, Farrukhabad, (U.P) with a chief complaint of swelling and pain in the right side of lower jaw since 4 months. (Figure 1) The swelling was insidious in onset and gradually progressive causing facial asymmetry. It was associated with mild pain. The clinical findings suggested an impacted 43 which was attached and embedded to the lingual cortical plate. The cystic lumen was filled with keratin. Radiographic findings revealed radiolucency extending from 36 to the right ramus of the mandible. (Figure 2)Based on clinical and radiographic features, a provisional diagnosis of odontogenic keratocyst was given.

The operative procedure was performed for the same and during the surgical procedure, it was difficult to remove the impacted tooth since there was a risk of fracture. The obtained tissue specimen was sent to the Department of Oral and Maxillofacial Pathology, Sri Rajiv Gandhi College of Dental Sciences and Hospital, Cholanagar, Hebbal, Bangalore, Karnataka, India for histopathological examination to rule out an OKC or ameloblastoma.
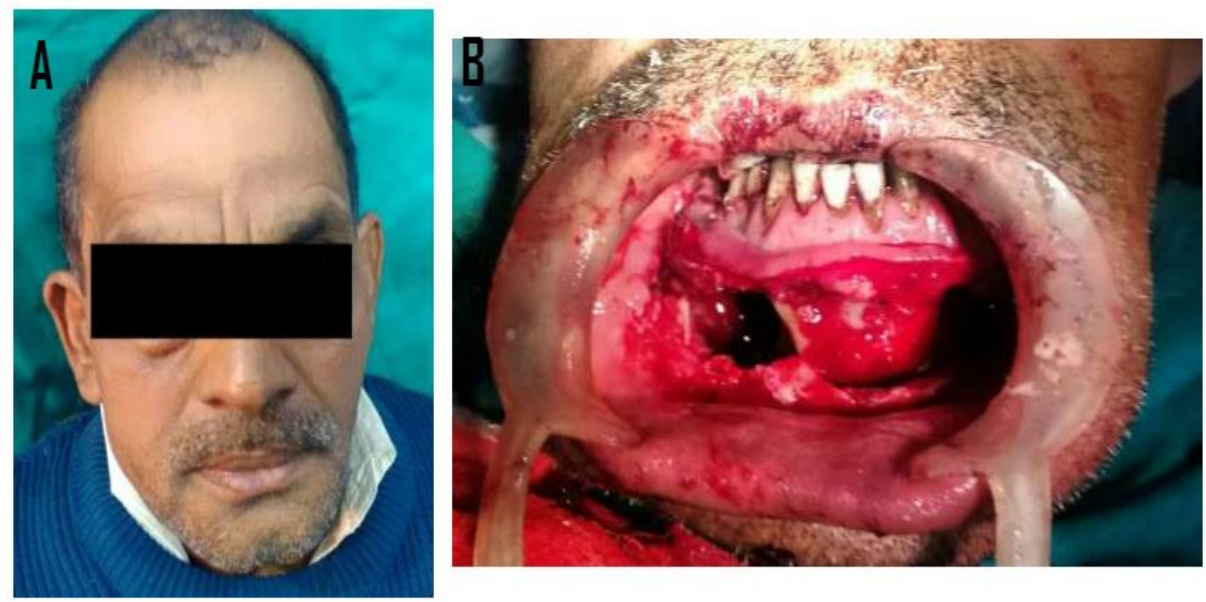

Figure 1.(A) Showing asymmetry of the face. B) Surgical view.

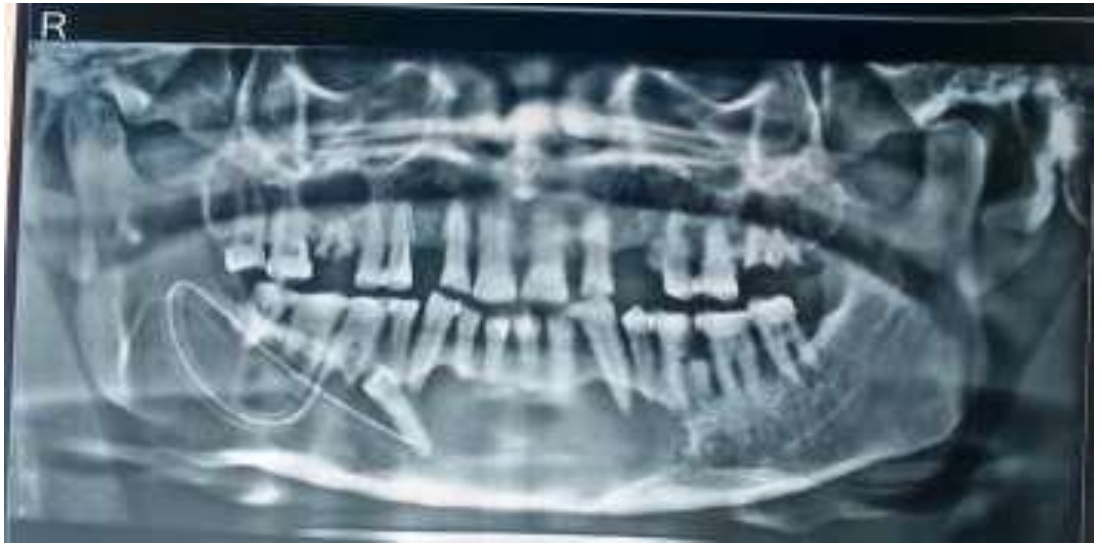

Figure 2.0PG revealing radiolucency extending from 36 to the right ramus of the mandible. 


\section{Histopathological Findings}

The H\&E stained soft tissue section shows the cystic lining of 3-4 layers thick epithelium with fibrous connective tissue capsule. (Figure 3) The epithelium shows prominent granular cell layer showing keratinization. The transition of epithelium seen from reduced enamel epithelium to keratinized epithelium. Areas of epithelial cell proliferation are also seen along with inflammation at focal areas. Keratin flakes are seen in the lumen. Underlying connective tissue shows moderate collagen fibers interspersed with blood vessels.

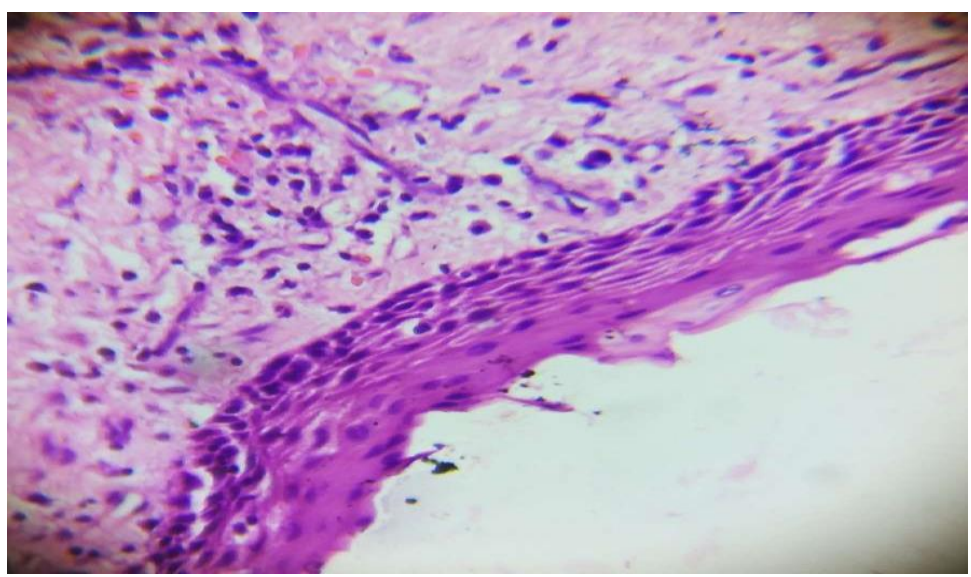

Figure 3.H \& E stained section showing cystic lining with a thickness of 3-4 cell layers overlying the fibrous connective tissue capsule. (10x).

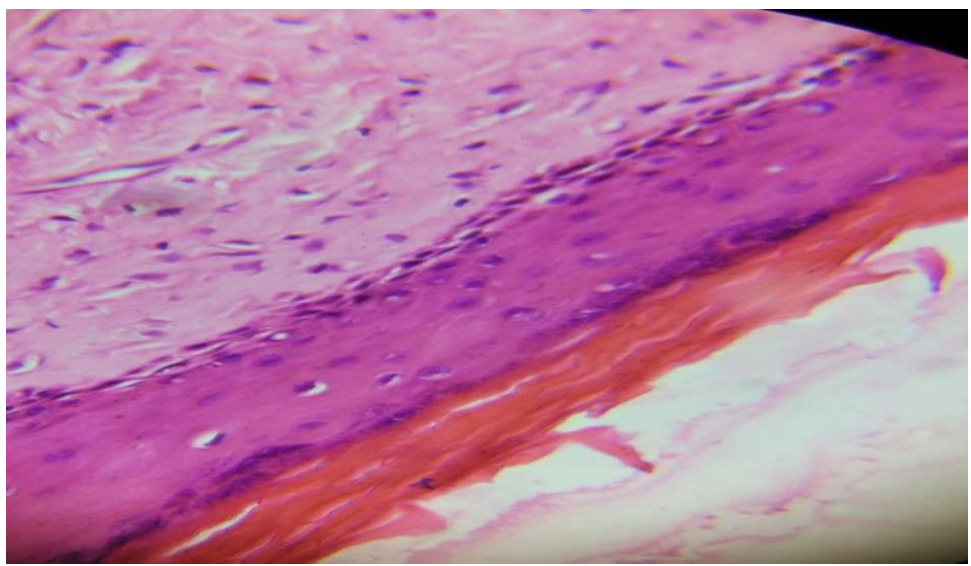

Figure 4.H \& E Stained section showing the keratinized lining epithelium with areas of the prominent granular cell layer. (40x).

\section{Discussion}

The dentigerous cyst was first of all described in France in 1778. Harris C.A 1842 was the first to publish a case report on the dentigerous cyst. Benn 1991 stated that inflammation at the apex of the deciduous tooth can lead to the development of an inflammatory follicular cyst. ${ }^{[4]}$ An odontogenic cyst is most commonly seen between 10 to 30 years of age and may remain asymptomatic throughout life. In such cases, it is usually diagnosed incidentally on a skull or tooth radiograph, if it is not treated properly it can lead to pathological fracture or get secondarily infected. ${ }^{[2]}$

Philipsen, 1956suggested the term Odontogenic Keratocyst (OKC) for all Odontogenic cysts, regardless of type, showing keratinization of the epithelium. Recently, an OKC is defined by other characteristics of the epithelium such as basal palisading, hyperchromatic nuclei and cell thickness of the epithelium and 
not merely the presence of keratinization. The term "keratinizing odontogenic cyst" has been suggested for any cyst, regardless of the type, that shows keratinization. ${ }^{[1]}$

Gold, 1963 introduced the terms keratinizing and calcifying odontogenic cysts. The epithelial lining of a dentigerous cyst is not keratinized commonly and characteristically when it is keratinized it is related to OKC. $[8]$

The increased osmolarity of the cyst fluid increases the internal hydrostatic pressure of the cyst. The connective tissue of the cyst particularly adjacent to the epithelium contains mast cells which due to metabolic turnover and inflammatory degradation release heparin sulphate, glycosaminoglycans and proteoglycans. Most of the dentigerous cyst show acute and chronic inflammation in their walls. ${ }^{[9]}$

Arendorg, 1981 stated the role of prostaglandins in the expansion of dentigerous cyst. He stated that PGE2 and PGE3 which are released from the dental follicle may resorb cementum and dentine which in turn causes expansion of the cyst.[9]

Meghji, Harvey, Harris in 1989 stated that the monocyte-macrophage infiltrate along with the stromal fibroblasts release interleukin 1 which causes a number of osteolytic cell reactions, stimulating osteoclasts and resorption of bone stimulating connective tissue cells which in turn causes the production of collagenase which in turn leads to the destruction of the bone matrix.[10]

Histologically, when dentigerous cysts are not infected are lined by a layer of non-keratinized stratified squamous epithelium without rete ridge formation with a surrounding wall of thin connective tissue containing odontogenic epithelial rests with glycosaminoglycan ground substance. ${ }^{[4]}$

When dentigerous cysts are inflamed the epithelial lining is thicker along with rete pegs formation. Wall of inflamed dentigerous cysts is more densely collagenized. Focal mucin-producing cells i.e mucous cells are often found in the epithelial lining. Rarely the ciliated epithelial cells may be found. Sebaceous cells may be noted within the fibrous cyst. Mucous, ciliated and sebaceous elements represent the multipotentiality of the odontogenic epithelial lining. Immuno-histochemical study shows higher IgG plasma cells and the lower percentage of IgA containing plasma cells in dentigerous cysts than in other cysts especially keratocyst. ${ }^{[4]}$

Clinical examinations, tooth vitality tests, FNAC, radiographs, biopsy, and ultrasounds are some of the important diagnostic tools which are being used on a regular basis. Radiologically differential diagnosis includes follicular spaces, Unicystic ameloblastomas, AOT, ameloblastic fibromas and OKC.[11]

The treatment for small dentigerous cysts includes enucleation and removal of the impacted tooth or tooth left with cyst enucleation and made to erupt. Whereas, in cases of the large dentigerous cysts marsupialization is performed which permits decompression of the cyst with a reduction in the bone defect. The prognosis is excellent while recurrence is rare. However, long term follow-up in subjects of keratinizing dentigerous cyst is usually employed to rule out its or malignant transformation and potential for recurrence. ${ }^{7]}$

Complications include ameloblastoma, Squamous cell carcinoma in about 1-2 \% of cases, odontogenic cysts in about $25 \%$ cases, intraosseous mucoepidermoid carcinomas arising from the mucous lining of the dentigerous cyst.[9]

\section{Conclusion}

Early diagnosis and treatment of odontogenic cyst lesions are very important to prevent morbidity. Knowledge of histopathologic features helps confirm the diagnosis and eases the treatment plan. Keratinizing dentigerous cyst is an unusual histological variant of an odontogenic cyst which is not seen very regularly in the clinical practice. Hence proper treatment plan and follow up is a necessity due to its extreme malignant transformation and potential for recurrence. 


\section{References}

1. Sivasankar V, Ranganathan K, Praveen B. Keratinizing dentigerous cyst. Contemp Clin Dent 2014;5:127-9.

2. Di Pasquale $P$, ShermetaroC. Endoscopic removal of a dentigerous cyst producing unilateral maxillary sinus opacification on computed tomography. Ear Nose Throat J.2006; 85:747-8.

3. Szerlip L. Displaced the third molar with a dentigerous cyst--An unusual case. J Oral Surg 1978;36:551-2.

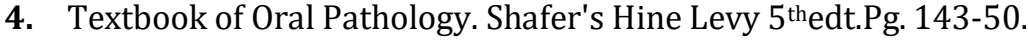

5. Elango S, Palaniappan SP. An ectopic tooth in the roof of the maxillary sinus. Ear Nose Throat J 1991;70:365-6.

6. Ramakrishna A, Lambade P. Dentigerous cyst associated with ectopic canine and a supernumerary tooth: A rare occurrence. J Surg Tech Case Report 2013; 5:85-8.

7. Mar and Stern Oral and Maxillofacial Pathology, $1^{\text {st }}$ edt.2003.Pg.35-41.

8. Samartano JG, Haar JG. A large keratinizing dentigerous cyst:Report of a case. J Oral Surg 1971; 29:60-62.

9. Oral and Maxillofacial Pathology Neville Damm Allen Bouquot $2^{\text {nd }}$ edt. Pg. 189-97.

10. Jeyaraj p, Mohan SM. Dentigerous cyst of the mandible with multiple complex odontomas. MJAFI.2008; 64:382-3.

11. Farahani SS, Lotfalian M. A pigmented dentigerous cyst in a patient with multiple dentigerous cysts of the jaws: a case report. J Contemp Dent Pract. 2007; 8:85-91.

How to cite this Article: Sarvesh Vijay, Yogesh T.L, Dr.Anjani K Jha, Nonitha.S, Keratinizing Dentigerous Cyst - An Unusual Entity-A Rare Case Report

Int. J. Drug Res. Dental Sci., 2019; 1(1): 6-10.

Source of Support: Nil, Conflict of Interest: None declared.

\section{Received: 08-02-2019 Revised: 04-03-2019 Accepted: 12-03-2019}

